\title{
Comportamentos de risco para transtornos do comportamento alimentar e fatores associados entre estudantes de nutrição do município do Rio de Janeiro
}

\section{Risk behavior for eating disorders and related factors among nutrition students in the city of Rio de Janeiro}

\author{
Maria Lucia Magalhães Bosi, Ronir Raggio Luiz, Caroline Maia da Costa Morgado, \\ Mara Lucia dos Santos Costa, Rosemary Jane de Carvalho
}

\begin{abstract}
Resumo
Objetivo: Caracterizar práticas alimentares e fatores de risco associados a transtornos do comportamento alimentar entre estudantes de nutrição do município do Rio de Janeiro. Métodos: Estudo seccional junto a um segmento populacional apontado na literatura como de risco para o surgimento de transtornos alimentares. Utilizaram-se o Teste de Investigação Bulímica de Edimburgo (BITE), o Teste de Atitudes Alimentares (EAT-26) e uma variável que considera os dois instrumentos associados (Nunes et al., 2001). Resultados: Analisaram-se 193 estudantes do sexo feminino, com média de idade de 20,9 anos \pm 2 anos. Detectou-se resultado positivo em $14 \%$ (intervalo de confiança [IC] 95\%: 9,4\%-20\%) no EAT-26. No BITE, para sintomas elevados e gravidade intensa, foram encontradas prevalências de 5,7\% (IC 95\%: 2,9\%-10\%) e 3,2\% (IC 95\%: 1,2\%-6,9\%), respectivamente. Quando combinados EAT-26 positivo e BITE com gravidade intensa e sintomas elevados, constataram-se correlações positivas com prevalências de $64,7 \%(p<0,001)$ e $36,4 \%(p<0,001)$, respectivamente. Das mulheres que apresentaram EAT-26 positivo, 88,5\% encontram-se na faixa de normalidade do índice de massa corporal (IMC) $(p<0,031)$. Conclusões: Deve-se atentar para comportamentos de risco para transtornos alimentares no grupo, uma vez que esses distúrbios serão objeto de sua prática profissional, podendo comprometê-la nos casos em que nutricionistas sejam portadores de síndromes instaladas ou comportamentos precursores. Palavras-chave: transtornos do comportamento alimentar, universitárias, psiquiatria, saúde mental.
\end{abstract}

\begin{abstract}
Objective: To characterize risky eating habits and factors related to eating disorders among nutrition students in the city of Rio de Janeiro Methods: Sectional study with a segment of the population pointed out in literature as being at risk of developing eating disorders. The Bulimic Investigatory Test Edinburgh (BITE) questionnaire, the Eating Attitudes Test (EAT-26) questionnaire and a variable which considers the two related tools (Nunes et al, 2001) were used. Results: One hundred ninety-three female students were studied, being 20.9 years old on the average \pm 2 years. A positive result of 14\% (IC 95\%: 9.4\%-20\%) in the EAT-26 was observed. In the BITE advanced symptoms and scores in the serious range were found in 5.7\% (IC 95\%: 2.9\%-10\%) and $3.2 \%$ (IC 95\%: 1.2\%-6.9\%), respectively. When positive EAT-26 results were combined with serious range BITE scores and advanced symptoms, positive correlations were found in $64.7 \%(p<0.001)$ and $36.4 \%(p<0.001)$, respectively. Of the women who showed positive EAT-26, $88.5 \%$ were within the normal range of the body mass index (BMI) $(p<0.031)$. Conclusions: One must pay close attention to the tendencies towards eating disorders in the group, since these disorders will be a focus of their professional practice, which could compromise their work in the cases where nutritionists already present syndromes or tendencies towards disorders.

Key words: eating disorders, university students, psychiatry, mental health.
\end{abstract}

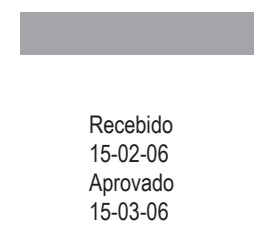

Núcleo de Estudos em Saúde Coletiva (NESC) da Universidade Federal do Rio de Janeiro (UFRJ) (Bosi MLM); NESC/UFRJ (Luiz RR, Morgado CMC, Costa MLS, Carvalho RJ)

Trabalho financiado pelo Conselho Nacional de Desenvolvimento Científico e Tecnológico (CNPq), Edital Universal 2003, e pela Fundação José Bonifácio - Programa de Apoio à Pesquisa.

Correspondência para: Maria Lucia M. Bosi - Núcleo de Estudos de Saúde Coletiva da UFRJ -NESC

Pça da Prefeitura da Cidade Universitária - Av. Brigadeiro Trompowski, s/nº - CEP 21949-900 - Rio de Janeiro-RJ Tels: (21) 2598-9328 / 2598-9271 - e-mail: malubosi@nesc.ufrj.br 


\section{Introdução}

$\mathrm{Na}$ atualidade, no contexto das sociedades ocidentais, o culto à beleza está diretamente associado à imagem de poder e mobilidade social, dentro de um contexto fortemente contraditório e paradoxal: por um lado, movimentam-se os lucros das indústrias de alimentos hipercalóricos, via incentivo ao consumo, e, por outro, cobra-se sujeição a um ideal estético cada vez mais difícil de atingir e notadamente conflitante com o consumo alimentar incentivado. Nesse cenário, emergem os transtornos do comportamento alimentar como questão social e de saúde pública, sobretudo junto à população feminina jovem (Cordás e Castilho, 1994; Nunes et al., 2001; Fiates e Salles, 2001).

Embora haja discordância no que se refere ao aumento da prevalência (Hay, 2002), a literatura especializada conflui no sentido de apontar o sexo feminino como o mais vulnerável às pressões socioeconômicas e culturais associadas aos padrões estéticos, tendo-se em vista a ênfase crescente dada à magreza como aspecto associado aos ideais de beleza. Ante essa situação, que acarreta forte discriminação contra 0 corpo obeso, expressando o que alguns autores nomeiam de lipofobia, as mulheres que se encontram insatisfeitas com 0 seu corpo utilizam, para controle do peso corporal, uma gama de recursos como dietas fortemente restritivas ou modismos dietéticos, exercícios excessivos, uso de laxantes, diuréticos e drogas anorexígenas, entre outros recursos que dissociam estética e saúde (Bosi e Andrade, 2004; Fiates e Salles, 2001; Dunker e Philippi, 2003).

Essas práticas e comportamentos, considerados síndromes parciais, são os principais fatores de risco para o desenvolvimento dos chamados transtornos do comportamento alimentar (TCA), que correspondem aos diagnósticos de anorexia nervosa, bulimia nervosa e transtornos alimentares não-específicos, incluindo-se neste, como categoria ainda em estudo, o transtorno da compulsão alimentar periódica (TCAP) (Appolinário, 1998). Tais modalidades de comportamento, inscrevendo-se entre os transtornos mentais, acarretam uma série de sintomas e seqüelas, chegando mesmo, em certos casos, a comprometer a sobrevivência dos seus portadores, tendo-se em vista sua alta letalidade, como é o caso da anorexia.

A anorexia nervosa é caracterizada por uma restrição alimentar auto-imposta com seqüelas graves, características obsessivo-compulsivas e simbolizações que, freqüentemente, tornam complexo o tratamento. 0 episódio bulímico refere-se à ingestão de uma quantidade exagerada de alimentos (binge), comportamento que pode ocorrer eventualmente em indivíduos normais, sendo que a bulimia não visa apenas a saciar uma fome exagerada, mas atende a uma série de estados emocionais eliciados por situações estressantes, incluindo a oportunidade de purgação (Fiates e Salles, 2001; Cordás e Claudino, 2002; Herscovici, 1997).

Já a descrição do TCAP surgiu pela necessidade de diferenciar indivíduos obesos com compulsão daqueles sem compulsão alimentar e dos bulímicos. No diagnóstico desse transtorno inserem-se episódios recorrentes de compulsão alimentar que envolvem características como o excesso alimentar e a perda de controle. Ressalta-se que os pacientes com TCAP apresentam dificuldades para circunscrever os episódios de compulsão alimentar, em virtude de não apresentarem, contrariamente aos bulímicos, engajamento em métodos compensatórios subseqüentes ao mesmo (Claudino e Borges, 2002; Herscovici, 1997).

Cabe acrescentar, ainda, que a anorexia e bulimia nervosas, embora classificadas separadamente, estão intimamente relacionadas por apresentarem psicopatologia comum: um padrão recorrente envolvendo a preocupação excessiva com o peso e com a forma corporal (Wiseman, 1988; Claudino e Borges, 2002). Uma revisão sistemática de 12 estudos de incidência cumulativa realizada por Pawluck e Gorey (1998) relatou uma incidência média anual de anorexia nervosa na população, em geral, de 18,5 por 100 mil entre mulheres e de 2,25 por 100 mil por ano entre homens. Já a incidência estimada de bulimia nervosa foi mais elevada, sendo de 28,8 entre muIheres e de 0,8 em homens por 100 mil a cada ano. Uma vez instalados, esses quadros são muito resistentes a tratamento, o que reforça a necessidade de intervenções de caráter preventivo e as realizadas por equipe multidisciplinar formada por nutricionistas, psicólogos, psiquiatras, endocrinologistas, entre outros profissionais.

No que se refere aos profissionais de nutrição, focalizados neste estudo, assinalamos que, até anos recentes, a temática dos transtornos alimentares não constituía um campo sistematicamente trabalhado pela categoria, porém esse profissional representa uma parte importante na assistência interdisciplinar e multidimensional recomendada para a assistência aos transtornos alimentares, conforme a American Dietetic Association (ADA) (Alvarenga, Larino, 2002; Cordás, 2001). Por um lado, pouco se sabe acerca de comportamentos alimentares e práticas de controle de peso nessa categoria, sendo escassos os estudos na literatura consultada, embora já se localizem iniciativas, algumas delas ainda em desenvolvimento. A literatura aponta indícios de uma prevalência aumentada em certos grupos profissionais que lidam com aspectos relacionados a nutrição e saúde (Nunes et al., 2001; Fiates e Salles, 2001). Sendo assim, esta investigação visa a contribuir para o dimensionamento do problema nesse segmento específico através da caracterização das práticas alimentares e outros fatores associados a TCA entre estudantes de Nutrição no município do Rio de Janeiro.

\section{Métodos}

O presente estudo integra o projeto de pesquisa Comportamentos Alimentares Anormais e Práticas Inadequadas de Controle de Peso entre Estudantes Universitários, de delineamento transversal, em desenvolvimento no âmbito de uma universidade pública situada no município do Rio de Janeiro, abrangendo seis cursos de graduação: medicina, educação física, psicologia, nutrição, letras e engenharia. A escolha desses cursos deveu-se ao fato de serem os quatro primeiros aqueles que formam profissionais inseridos nas especialidades que seriam mais procuradas pelos portadores de TCA e de síndromes parciais, sendo, portanto, categorias diretamente envolvidas no manejo clínico dos referidos agravos. Quanto às duas últimas carreiras, sua inclusão se deu para que se 
pudesse contemplar um contraste no que se refere às variáveis sexo e inserção na área da saúde, aspectos que poderiam se expressar nos resultados obtidos.

Para calcular o tamanho da amostra no curso de nutrição, na ausência de estudos que definissem a prevalência do problema nesse segmento específico, estimou-se em 10\% a chance de alguma das alunas apresentar comportamentos alimentares anormais ou práticas inadequadas de controle de peso, como proposto por Nunes (1997) em estudo realizado em Porto Alegre, incluindo essa faixa, considerando-se de $4 \%$ a margem de erro aceitável. Para populações grandes e estabelecendo-se uma confiança de $95 \%$, obtém-se uma estimativa do tamanho amostral de 189 indivíduos por segmento. Entretanto o total de alunos do curso de nutrição foi de, aproximadamente, 300, o que nos levou a adotar como estratégia investigar todo o curso.

A amostra efetivamente pesquisada foi de 220 universitários, porém optou-se por excluir os homens da análise aqui desenvolvida, uma vez que representavam um número pequeno no curso $(n=27)$, além de caracterizar uma dimensão aparentemente não-resgatável pelo instrumento, dado 0 viés de sexo dos instrumentos utilizados (Melin e Araújo, 2002), que compromete suas sensibilidade e especificidade, exigindo algumas mediações ao se comparar esses resultados com os achados relativos ao grupo feminino. A análise dos dados referentes aos informantes do sexo masculino dos cursos investigados encontra-se ainda em desenvolvimento e será efetuada com base na estratégia de triangulação metodológica, de modo a conferir maior rigor (Bosi e Mercado, 2004). Assim, com base na opção de considerar, primeiramente, os resultados referentes ao grupo feminino, finalizamos com uma amostra de 193 alunas, junto à qual se aplicaram os inventários comentados a seguir, além de um instrumento por meio do qual foram levantadas características pessoais e sociais do grupo.

Os instrumentos utilizados para avaliação dos comportamentos alimentares anormais e das práticas inadequadas de controle de peso foram o Teste de Atitudes Alimentares (EAT-26) (Garner e Garfinkel, 1979), em sua versão em português (Nunes et al., 1994), e o Teste de Investigação Bulímica de Edimburgo (BITE) (Henderson e Freeman, 1987), também em sua versão para o português (Cordás e Hochgraf, 1993). O instrumento EAT-26 mede, principalmente, comportamentos alimentares restritivos, como dieta e jejum, e comportamentos bulímicos, como ingestão excessiva de alimentos e vômitos provocados. Dependendo do tipo de resposta às 26 questões do teste, um escore é calculado, e pessoas com mais de 20 pontos são classificadas como apresentando comportamentos alimentares sugestivos de anormalidade. O BITE avalia, predominantemente, comportamentos bulímicos, sendo um instrumento composto por 33 questões, com duas subescalas: de sintomas e de gravidade. A escala de sintomas classifica em três grupos de escores: elevado (maior ou igual a 20 pontos), médio (10 a 19 pontos) e normal (abaixo de 10 pontos). A escala de gravidade é utilizada quando o escore de sintomas é superior a 10 pontos e classifica os sintomas em três grupos, segundo a importância: intensa (escore de 10 ou mais), significativa (escore de 5 a 9) e sem gravidade (escore menor que 5).
Adotando-se a associação proposta por Nunes et al. (2001), foi construída uma variável que considera os dois instrumentos, EAT-26 e BITE, que se complementam na avaliação dos comportamentos alimentares, categorizando a amostra em três grandes grupos, conforme os critérios a seguir:

- comportamento alimentar anormal: mulheres com escores do BITE elevados para sintomas e escores intensos para gravidade;

- comportamento alimentar de risco: mulheres com escore do BITE médio para sintomas ou maior que 20 para o EAT-26; - comportamento alimentar normal: mulheres com escore do BITE normal para sintomas e escore menor ou igual a 20 pontos para o EAT-26.

Utilizou-se, ainda, como indicador antropométrico o índice de massa corporal (IMC), calculado a partir das variáveis peso e altura, informados (atual) e desejados (ideal). A classificação foi feita a partir da Organização Mundial da Saúde (OMS) (WHO, 1995).

A descrição geral dos dados será apresentada por meio de freqüências simples e relativas, adotando-se para a análise a inter-relação das variáveis selecionadas para a realização do teste qui-quadrado, utilizando-se para armazenamento e análise dos dados o programa SPSS 11.0.

\section{Resultados}

Das 193 estudantes de nutrição, a distribuição da faixa etária variou dos 17 aos 32 anos, sendo a média de idade 20,9 anos \pm 2 . A maioria das estudantes $(92,2 \%)$ é natural do estado do Rio de Janeiro, sendo que $51,3 \%$ dos seus pais possuem nível superior completo.

A prática de atividades físicas, caracterizada pela resposta positiva quanto à freqüência, prevaleceu, na amostra, com $54,4 \%$ das estudantes pertencendo a esse grupo. Entre as que praticam alguma atividade física, 19,7\% exercitam-se durante duas horas por dia.

Em relação à presença de algum familiar obeso, 69,4\% das universitárias responderam negativamente. A realização de algum tratamento estético ou cirurgia plástica devido a motivos estéticos foi constatada em $15,6 \%$ das estudantes. 0 estudo antropométrico baseou-se nos dados referidos pelas alunas, não tendo sido possível efetuar a avaliação antropométrica direta. A média de altura correspondeu a 1,64m $\pm 0,07$ e a de peso informado a $55,8 \mathrm{~kg} \pm 8,6$. A média do IMC foi $20,8 \mathrm{~kg} / \mathrm{m}^{2} \pm 2,5$, sendo considerado adequado pela classificação da OMS (WHO, 1995), cabendo assinalar que esse valor tende para o limite inferior. Para explorar a satisfação com o peso, solicitou-se que fosse respondido qual o peso desejado, tendo sido constatada uma insatisfação com o mesmo, pois a diferença entre o peso informado e o desejado foi de $2 \mathrm{~kg} \pm$ 4,5. Quando calculado o IMC com o peso desejado, seu valor foi $20,1 \mathrm{~kg} / \mathrm{m}^{2}$, o que reforça o desejo de atingir o padrão de beleza magro.

A análise dos instrumentos utilizados para detectar práticas de controle de peso inadequadas mostrou que 14\% das universitárias tiveram EAT-26 positivo, 5,7\% apresentaram sintomas elevados no BITE e $3,2 \%$ possuíam gravidade intensa no BITE (Tabela 1). 
De acordo com a categorização criada, foi observado que $67,4 \%$ das estudantes apresentavam comportamento alimentar normal, 21,2\% o apresentavam de risco e 11,4\% possuíam comportamento alimentar anormal. Constata-se, portanto, que os comportamentos alimentares anormais apresentaram uma freqüência de $32,6 \%$ nessas universitárias (Tabela 2).

Quando associado o EAT-26 com as variáveis IMC, insatisfação com o peso e idade, observou-se que $88,5 \%$ das graduandas que apresentavam EAT-26 positivo tinham IMC adequado. Com relação ao IMC de sobrepeso/obesidade, $11,5 \%$ tinham EAT-26 positivo e 2,4\% o tinham normal $(p=0,031)$. Na associação entre a insatisfação com o peso e o EAT-26 foi encontrada uma diferença estatisticamente significativa $(p=0,001)$ entre as alunas que apresentaram o resultado do teste positivo e as que queriam perder até dois quilos ou mais ( $96 \%)$, se comparadas com as que apresentavam o teste normal e que também queriam perder peso $(60,1 \%)$. A associação entre EAT-26 e idade não apresentou diferença estatisticamente significativa $(p<0,452)$ (Tabela 3 ).

$\mathrm{Na}$ Tabela 4 descreve-se a freqüência percentual de alguns itens do questionário EAT-26, material aqui categorizado em duas dimensões: subjetiva (preocupação) e prática (ações), ou seja, respectivamente, aspectos que ainda se mantinham no plano da subjetividade e aqueles efetivamente já praticados pelo grupo. Constatou-se, assim, que, entre os aspectos analisados, há uma preocupação constante das alunas com o desejo de serem mais magras
Tabela 1. Escores no BITE e no EAT em estudantes de nutrição na cidade do Rio de Janeiro ( $n=193)$

\begin{tabular}{lccc}
\hline \multicolumn{1}{c}{ Escore } & $n$ & $\%$ & IC 95\%* \\
\hline $\begin{array}{l}\text { BITE - gravidade intensa } \\
\text { ( } \geq 10 \text { pontos) }\end{array}$ & 6 & 3,2 & $1,2 \%-6,9 \%$ \\
$\begin{array}{l}\text { BITE - sintomas elevados } \\
(\geq 20 \text { pontos) }\end{array}$ & 11 & 5,7 & $2,9 \%-10 \%$ \\
$\begin{array}{l}\text { EAT-26 positivo } \\
(\geq 21 \text { pontos) }\end{array}$ & 27 & 14 & $9,4 \%-20 \%$ \\
\hline
\end{tabular}

IC $95 \%$ = intervalo de confiança de $95 \%$.

Tabela 2. Freqüência percentual da categorização do comportamento alimentar de estudantes de nutrição na cidade do Rio de Janeiro

\begin{tabular}{lcc}
\hline Categorização & $n$ & $\%$ \\
\hline Anormal & 22 & 11,4 \\
Risco & 41 & 21,2 \\
Normal & 130 & 67,4 \\
Total & 193 & 100 \\
\hline
\end{tabular}

$(17,1 \%)$, além de $10,4 \%$ terem respondido que sempre têm o costume de fazer dieta. Já a preocupação de fazer exercício pensando em queimar calorias atinge sempre $26,8 \%$ das estudantes.

Tabela 3. Pontuações do EAT-26 segundo algumas características demográficas e clínicas da amostra $(n=193)$

\begin{tabular}{|c|c|c|c|c|c|c|c|}
\hline \multirow[b]{3}{*}{ Características } & & & \multicolumn{4}{|c|}{ EAT-26 } & \multirow{3}{*}{ Valor de $p^{*}$} \\
\hline & \multicolumn{2}{|c|}{ Total } & \multicolumn{2}{|c|}{ Até 20 pontos } & \multicolumn{2}{|c|}{21 ou mais } & \\
\hline & $n$ & $\%$ & $n$ & $\%$ & $n$ & $\%$ & \\
\hline \multicolumn{8}{|l|}{ Idade } \\
\hline 17 a 19 anos & 45 & 23,3 & 39 & 20,2 & 6 & 22,2 & \\
\hline 20 ou 21 anos & 75 & 38,9 & 67 & 40,4 & 8 & 29,6 & 0,452 \\
\hline 22 anos ou mais & 73 & 37,8 & 60 & 36,1 & 13 & 48,1 & \\
\hline Total & 193 & 100 & 166 & 100 & 27 & 100 & \\
\hline \multicolumn{8}{|l|}{ IMC $\left(\mathrm{kg} / \mathrm{m}^{2}\right)$} \\
\hline Até 17,99 & 12 & 6,3 & 12 & 7,3 & 0 & 0 & \\
\hline 18 a 24,99 & 171 & 90 & 148 & 90,2 & 23 & 88,5 & 0,031 \\
\hline 25 ou mais & 7 & 3,7 & 4 & 2,4 & 3 & 11,5 & \\
\hline Total & 190 & 100 & 164 & 100 & 26 & 100 & \\
\hline \multicolumn{8}{|l|}{ Insatisfação com o peso** } \\
\hline Querem ganhar peso & 37 & 19,7 & 37 & 22,7 & 0 & 0 & \\
\hline Satisfeitas & 29 & 15,4 & 28 & 17,2 & 1 & 4 & 0,001 \\
\hline Querem perder até 2kg & 52 & 27,7 & 45 & 27,6 & 7 & 28 & \\
\hline Querem perder mais de $2 \mathrm{~kg}$ & 70 & 37,2 & 53 & 32,5 & 17 & 68 & \\
\hline Total & 188 & 100 & 163 & 100 & 25 & 100 & \\
\hline
\end{tabular}

*Teste $X^{2 ;}$; ${ }^{* *}$ peso - peso ideal. 
Tabela 4. Distribuição das freqüências percentuais de resposta ao EAT-26

\begin{tabular}{|c|c|c|c|c|c|c|c|}
\hline \multirow[b]{2}{*}{$\begin{array}{c}\text { Itens selecionados do } \\
\text { EAT-26 }\end{array}$} & \multicolumn{6}{|c|}{ Freqüência percentual assinalada para cada item do EAT-26 } & \multirow[t]{2}{*}{ Média } \\
\hline & Sempre & $\begin{array}{c}\text { Muito } \\
\text { freqüente }\end{array}$ & Freqüentemente & $\begin{array}{c}\text { Algumas } \\
\text { vezes }\end{array}$ & Raramente & Nunca & \\
\hline \multicolumn{8}{|l|}{ Dimensão prática (ações) } \\
\hline 1. Costumo fazer dieta & $10,4 \%$ & $8,9 \%$ & $11,5 \%$ & $17,7 \%$ & $19,3 \%$ & $32,3 \%$ & 4,23 \\
\hline 15. Vomito depois de comer & $0 \%$ & $0,5 \%$ & $0,5 \%$ & $2,1 \%$ & $2,6 \%$ & $94,3 \%$ & 5,9 \\
\hline $\begin{array}{l}\text { 20. Corto minha comida em } \\
\text { pedaços pequenos }\end{array}$ & $13 \%$ & $6,2 \%$ & $10,4 \%$ & $31,1 \%$ & $15,5 \%$ & $23,8 \%$ & 4,02 \\
\hline $\begin{array}{l}\text { 21. Levo mais tempo que os } \\
\text { outros para comer }\end{array}$ & $12 \%$ & $9,9 \%$ & $8,4 \%$ & $29,3 \%$ & $17,8 \%$ & $22,5 \%$ & 3,98 \\
\hline $\begin{array}{l}\text { 25. Evito comer quando } \\
\text { estou com fome }\end{array}$ & $0,5 \%$ & $1,6 \%$ & $0,5 \%$ & $7,8 \%$ & $17,6 \%$ & $72 \%$ & 5,56 \\
\hline \multicolumn{8}{|c|}{ Dimensão subjetiva (preocupações) } \\
\hline $\begin{array}{l}\text { 7. Estou preocupada com o } \\
\text { desejo de ser mais magra }\end{array}$ & $17,1 \%$ & $11,9 \%$ & $11,9 \%$ & $24,9 \%$ & $13 \%$ & $21,2 \%$ & 3,68 \\
\hline $\begin{array}{l}\text { 9. Quando faço exercícios, } \\
\text { penso em queimar calorias }\end{array}$ & $26,8 \%$ & $11,1 \%$ & $9,5 \%$ & $24,2 \%$ & $9,5 \%$ & $18,9 \%$ & 3,35 \\
\hline $\begin{array}{l}\text { 10. Sinto-me extremamente } \\
\text { culpada depois de comer }\end{array}$ & $2,6 \%$ & $1 \%$ & $2,6 \%$ & $30,2 \%$ & $24,5 \%$ & $39,1 \%$ & 4,9 \\
\hline $\begin{array}{l}\text { 14. Tenho vontade de vomi- } \\
\text { tar após as refeições }\end{array}$ & $1 \%$ & $0,5 \%$ & $1 \%$ & $2,6 \%$ & $7,8 \%$ & $87 \%$ & 5,77 \\
\hline $\begin{array}{l}\text { 18. Acho-me uma pessoa } \\
\text { preocupada com comida }\end{array}$ & $11,9 \%$ & $11,4 \%$ & $18,7 \%$ & $25,9 \%$ & $18,1 \%$ & $14 \%$ & 3,69 \\
\hline
\end{tabular}

\section{Discussão}

Os achados referentes à prática de atividade física mostraram que quase metade da amostra foi inativa (45,6\%), o que corrobora outros resultados da literatura. Silva e Malina (2000) desenvolveram um estudo no sistema público de ensino de NiteróiRJ com adolescentes de 14 e 15 anos e encontraram que 41,6\% das meninas foram consideradas sedentárias. Oehlschlaeger et al. (2004), em um estudo na cidade de Pelotas-RS com adolescentes entre 15 e 18 anos, encontraram uma prevalência de sedentarismo no sexo feminino de $54,5 \%$. Podemos dizer que a inatividade física é resultante do estilo de vida moderno de trabalho e de lazer, caracterizado por atividades inertes, que se contrapõem ao desejo de ficar ou permanecer com o corpo magro ou mesmo se manter na faixa considerada normal, predispondo aos quadros de sobrepeso e obesidade, cuja importância como questão sociossanitária é cada vez mais preocupante, não só nos países industrializados, mas também em países periféricos como o Brasil.

Ao lado do aumento da inatividade física e do avanço da obesidade como problema de saúde pública, dados publicados pela Sociedade Brasileira de Cirurgia Plástica (SBCP), em 1999, já apontavam o aumento do número de adolescentes e jovens submetendo-se a operações plásticas por motivos estéticos, tendo o número duplicado entre os anos de 1997 e 1998 (Andrade, Bosi, 2003). A prevalência de estudantes que realizaram tratamento estético ou cirurgia plástica nesse estudo (15,6\%) pode expressar a influência exercida pela sociedade contemporânea nos padrões estéticos e no avanço da tecnologia da beleza, o que contribui também para alterações no comportamento alimentar. Contudo o método empregado não autoriza conclusões em relação a esse aspecto, uma vez que são necessários estudos fundamentados na abordagem qualitativa que possam aprofundar a compreensão dessas, entre outras dimensões aqui indicadas.

Conforme já assinalado, ainda são escassos os estudos com estudantes universitários a fim de estimar a prevalência de alterações no comportamento alimentar. Neste estudo, os resultados dos instrumentos utilizados mostraram que $14 \%$ das universitárias de nutrição tiveram EAT-26 positivo. Quando esse resultado é comparado com o estudo de Fiates e Salles (2001), com estudantes de nutrição da Universidade Federal de Santa Catarina (UFSC), 22,2\% foram classificadas com EAT-26 positivo. Um estudo semelhante a esse (Castro e Goldstein, 1995) encontrou percentual de $20 \%$, enquanto Pastore et al. (1996) encontraram $15 \%$ e Nunes et al. (2001), 16,6\% para o EAT-26 positivo, resultados similares aos desse estudo.

Na escala de sintomas do BITE, os resultados encontrados neste estudo foram $5,7 \%$ das estudantes com sintomas elevados e 3,2\% com gravidade intensa, enquanto em Nunes et al. (2001), 3,3\% apresentaram padrão alimentar severamente perturbado e presença de comer compulsivo. Esses achados sugerem que as estudantes de nutrição apresentaram percentual de EAT-26 positivo e BITE de sintomas elevados e gravidade intensa, de acordo com os resultados observados por outros autores, o que não deixa de ser preocupante. 
Ao compararmos os resultados da categorização criada para avaliar os comportamentos alimentares deste estudo com os de Nunes et al. (2001), vimos que a presença de comportamento alimentar anormal foi igual nos dois, sendo $11,4 \%$ e $11,3 \%$, respectivamente; enquanto o comportamento alimentar de risco foi menor no nosso estudo $(21,2 \%)$ e o comportamento alimentar normal foi maior $(67,4 \%)$, em comparação também com Nunes et al. (2001), que tiveram resultados de $30,2 \%$ e $58,5 \%$, respectivamente. Vale destacar que o estudo desenvolvido por Nunes et al. (2001) inclui adolescentes. Entretanto considerando o comportamento alimentar fora do normal, temos $32,6 \%$ das estudantes, o que se revela um achado significativo para futuras nutricionistas.

O IMC médio das estudantes foi $20,8 \mathrm{~kg} / \mathrm{m}^{2} \pm 2,5$. Na associação entre o EAT-26 positivo e o IMC, vimos que entre as estudantes que apresentavam EAT positivo, 88,5\% eram eutróficas e 11,5\%, obesas. Quando o EAT-26 positivo foi associado à insatisfação com 0 peso, verificou-se que $96 \%$ gostariam de perder dois ou mais quilos. A representação desses resultados num curso de graduação de nutrição aponta para um perfil de mulheres jovens que apresentam alterações do comportamento alimentar sem fecharem diagnóstico para anorexia nervosa, principalmente pelo elevado percentual de adequação de peso e pela presença de pessoas com peso elevado, além da grande insatisfação com o peso/corpo pelo desejo de emagrecer, o que leva as mulheres a adotarem práticas de redução de peso muitas vezes inadequadas.

O estilo de vida contemporâneo evidencia uma necessidade crescente de os indivíduos procurarem formas de se manterem nos padrões estéticos exigidos pela cultura em que, no que se refere ao corpo, prevalecem processos simbólicos funcionais a um mercado controlado por setores hegemônicos que ditam a maneira de ser aceito. Poucos estudos têm sido desenvolvidos focalizando mulheres jovens que são cobradas a permanecerem nos padrões impostos para profissionais de passarela, modelos e manequins, quando sua realidade material é bastante distante, 0 que, não raro, impõe doença e sofrimento.

\section{Referências}

Alvarenga M, Larino MA. Terapia nutricional na anorexia e bulimia nervosas. Rev Bras de Psiquiatr, 24(supl. 3): 39-43, 2002.

Andrade A, Bosi MLM. Mídia e subjetividade: impacto no comportamento alimentar feminino. Revista de Nutrição, 16(1): 117-25, 2003.

Bosi MLM, Andrade A. Transtornos do comportamento alimentar: um problema de saúde coletiva. Cadernos de Saúde Coletiva, XII(2): 197-202, 2004.

Bosi MLM, Mercado FJ. Pesquisa qualitativa de serviços de saúde. Petrópolis: Vozes; 2004.

Castro JM, Goldenstein S. Eating attitudes and behaviors of pre- and postpubertal females: clues to the etiology of eating disorders. Physiol Behav, 58(1): 15-23, 1995.

Claudino AM, Borges MBF. Critérios diagnósticos para os transtornos alimentares: conceitos em evolução. Rev Bras de Psiquiatr, 24(supl. 3): 7-12, 2002.

Córdas TA, Claudino AM. Transtornos alimentares: fundamentos históricos. Rev Bras de Psiquiatr, 24(supl. 3): 3-6, 2002.

Córdas TA, Hochgraf PB. O BITE: instrumento para avaliação da bulimia

\section{Conclusão}

O estudo em questão aponta que quase metade da população estudada não pratica atividade física - embora supere a prática de atividades em outros segmentos -, ainda se aproxima do estilo de vida da sociedade moderna e se contrapõe à necessidade de cultivar os padrões estéticos exigidos. Além disso, podese observar que uma grande parcela das universitárias apresenta comportamento alimentar fora do normal e deseja perder peso. Esse é um achado de grande relevância, uma vez que a análise do IMC associado ao comportamento alimentar mostra que esse desejo não corresponde ao corpo em suas dimensões objetivas, visto que as informantes situam-se na faixa de peso adequada para sua estatura. Os resultados sinalizam a necessidade de um olhar mais atento, pois a tendência parece acentuar cada vez mais as práticas errôneas de controle de peso.

A banalização dessas práticas é um problema atual referido por profissionais e educadores que trabalham para identificar os transtornos alimentares. Os achados deste estudo devem servir de alerta para a questão dos transtornos alimentares na formação de profissionais da saúde, no caso nutricionistas, pois essas sofrem influências socioculturais que podem ser um importante fator motivador da escolha profissional.

Outra questão importante refere-se à formação, uma vez que se constata a necessidade de um olhar diferenciado das instituições formadoras sobre os próprios alunos. 0 fato de um cuidador apresentar comportamento alimentar anormal pode dificultar sua capacidade de identificar esses casos nas outras pessoas, sobretudo quando se associa a falta de preparo e o desconhecimento quanto a gênese, diagnóstico e manejo dos transtornos alimentares. Esses aspectos devem constituir objeto de outros estudos, já que evidenciam a necessidade ao mesmo tempo em que podem subsidiar uma revisão no processo de formação dos universitários, em especial na área da saúde. nervosa - versão para o português. J Bras Psiquiatr, 42: 141-4, 1993.

Cordás TA, Castilho S. Imagem corporal nos transtornos alimentares: instrumento de avaliação: Body Shape Questionnaire. Psiquiatria Biológica, 2(1): 17-21, 1994.

Cordás TA. Transtornos alimentares em discussão. Rev Bras de Psiquiatr, 23(4): 178-9, 2001.

Dunker KLL, Philippi ST. Hábitos e comportamentos alimentares de adolescentes com sintomas de anorexia nervosa. Revista de Nutrição, 16(1): 51-60, 2003.

Fiates GMR, Salles RK. Fatores de risco para o desenvolvimento de distúrbios alimentares: um estudo em universitárias. Revista de Nutrição, 14(supl.): 3-6, 2001.

Garner D, Garfinkel, P. The Eating Attitudes Test: an index of the symptoms of NA. Psychol Med, 9: 273-9, 1979.

Hay P. Epidemiologia dos transtornos alimentares: estado atual e desenvolvimentos futuros. Rev Bras Psiquiatr, 24(supl. 3): 13-7, 2002. 
Henderson M, Freeman CPL. A self-rating scale for bulimia. The BITE. Br J Psychiatr, 20: 18-24, 1987.

Hersovici CR. A escravidão das dietas. Tradução de Francisco Settineri. Porto Alegre: Artes Médicas; 1997.

Melin P, Araújo AM. Transtornos alimentares em homens: um desafio diagnóstico. Rev Bras Psiquiatr, 24(supl. 3): 73-6, 2002.

Nunes MA. Prevalência de comportamentos alimentares anormais e práticas inadequadas de controle de peso em mulheres de 12 a 21 anos em Porto Alegre. 1997. Mestrado [Dissertação]. Universidade Federal de Pelotas.

Nunes MAA, et al. O teste de atitudes alimentares (EAT-26) em adolescentes de Porto Alegre. Arquivos de Psiquiatria, Psicoterapia e Psicanálise, 1: 16-9, 1994

Nunes MA, Olinto MTA, Barrosa FC, Camey S. Influência da percepção do peso e do índice de massa corporal nos comportamentos alimentares anormais. Rev Bras Psiquiatr, 23(1): 21-7, 2001.

Oehlschlaeger MHK, et al. Prevalência e fatores associados ao sedentarismo em adolescentes de área urbana. Rev Saúde Pública, 38(2): 157-63, 2004.

Pastore DR, Fischer M, Friedman SB. Abormalities in weight status, eating attitudes and eating behaviors among urban high school students: correlations with self-esteem and anxiety. J Adolesc Health, 18(5): 312-9, 1996

Pawluck DE, Gorey KM. Secular trends in the incidence of anorexia nervosa: integrative review of population-based studies. Int J Eat Disord, 23: 347-52, 1998.

Silva RCR, Malina RM. Nível de atividade física em adolescentes do município de Niterói, Rio de Janeiro, Brasil. Cad de Saúde Pública, 16(4): 1091-7, 2000.

WHO - World Health Organization. Physical Status: the use and interpretation of anthropometry. Geneva, 1995. WHO Technical Report Series, $n^{\circ}$ 854.

Wisemam CV, Harris WA, Halmi KA. Eating disorders. Med Clin North Am, Philadelphia, 82(1): 145-59, 1988. 